\title{
Penetration of Supernova Ejecta in the Solar System
}

\author{
T. Athanassiadou* and B.D. Fields \\ Departments of Physics and Astronomy, \\ University of Illinois and Urbana-Champaign \\ E-mail: athanssdeillinois.edu, bdfields@illinois.edu
}

\begin{abstract}
In this paper, we investigate the mechanism by which supernova ejecta can penetrate the solar system, and in particular, directly deposit live radioactivities on Earth. A purely hydrodynamic interaction between a supernova blast and the solar wind yields a limit of $10 \mathrm{pc}$ as the maximum supernova explosion distance in order for supernova plasma to penetrate within 1AU. However, there exists evidence that the vast majority of heavy elements in a supernova remnant may be depleted onto grains, hence they can be considered as charged particles which do not participate in the plasma dynamics of the interaction of the supernova plasma and the solar wind. We examine the motion of these charged particles as they decouple from the supernova plasma and are influenced by the solar magnetic, radiation and gravitational field. We find that given the large incoming velocities of the charged grains, they suffer little or no deflection within the solar system. Consequently, the dust penetration to $1 \mathrm{AU}$ has essentially $100 \%$ transmission probability, and the dust capture onto the earth should have a geometric cross section.
\end{abstract}

10th Symposium on Nuclei in the Cosmos

July 27 - August 12008

Mackinac Island, Michigan, USA

* Speaker. 


\section{Introduction}

The possible geological signatures and biological consequences of supernova explosions near Earth have been a subject of great interest for almost half a century ([1, 2, 3]. Ellis et. al. [4] identified radioisotopes of intermediate (Myr) half-lives that could provide geological signatures of nearby, recent supernovae, while others examined the climatic and biological effects on Earth $[5,6]$. It was not until recently, though, that the subject moved from the realm of speculation to observational fact. Knie et. al. [7, 8] reported the discovery of live undersea ${ }^{60} \mathrm{Fe}$ in a ferromanganese crust in the Pacific Ocean. The signal was consistent with the ${ }^{60} \mathrm{Fe}$ being produced in a supernova that exploded 20-120 pc away [9] 2.8 Myrs ago.

One then needs to examine the mechanism by which supernova ejecta can get directly deposited on Earth. One method involves the supernova plasma overwhelming the solar wind, thus engulfing the Earth in ejecta. The maximum distance for such a scenario was shown to be $10 \mathrm{pc}$ [10], which is incidentally the distance for which detectable biological damage would occur [6].

Another method which is not only more favorable, but more realistic, concerns the delivery of supernova radioactivities in the form of freshly synthesized dust. There exist ample evidence that refractory heavy elements such as iron are depleted onto dust grains [11] and that Type II supernova are to some extent dust factories [12]. Perhaps the most convincing evidence is the discovery of extinct ${ }^{44} \mathrm{Ti}$ in presolar grains [13]. It remains controversial what fraction of supernova refractories remain in the dust phase throughout the life of the remnant [14] For the purposes of this paper, we will assume that some supernova dust participates in the remnant-heliosphere collision, and we examine the consquences for the deposition of supernova dust on the Earth.

\section{Supernova Plasma Delivery}

This section summarizes work which appears in Fields et. al. [10].

\subsection{Supernova Collision with the Solar Wind}

We simulated the solar wind, the supernova remnant and their collision using the FLASH code [15], an adaptive mesh, parallel simulation code. For the solar wind, we consider a spherically symmetric, adiabatic flow, where the solar wind speed is constant. The initial conditions were chosen so that they reproduce mean observed values for the solar wind speed, density and thermal pressure at $1 \mathrm{AU}$. For the supernova remnant evolution we adopt the spherically symmetric, adiabatic Sedov-Taylor solution, which is a good approximation to "nearby" supernovae that would not have entered the radiative phase. We considered explosions into interstellar media of various densities. For the collision over the relevant lenghtscales of AU's, the remnant was treated as a planar wind of uniform density, speed and pressure, consistent with the Sedov-Taylor solution for supernovae exploding at various distances from the solar system.

The interaction between the supernova remnant and the solar wind was treated purely hydrodynamically, i.e. we ignore the effects of magnetic fields.

\subsection{Results}

An illustrative example of the supernova-solar wind collision can be seen in Fig. 4. Since both the solar wind and supernova wind are supersonic, the interaction results into two shocks 

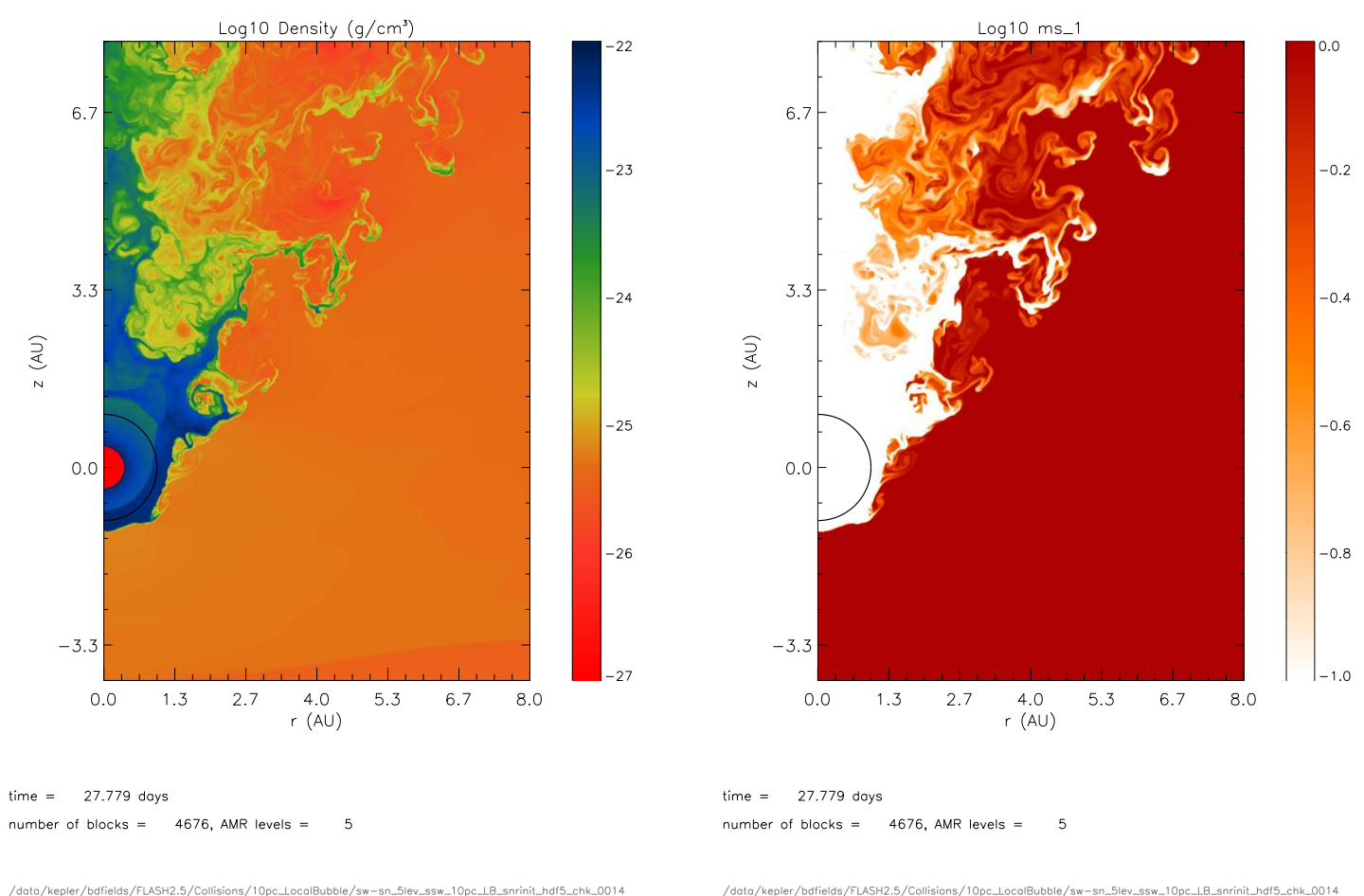

Figure 1: Left: Density map of SN-SW collision, for SN 10 pc away, exploding within the Local Bubble. Right: Contamination map of the same region. Red denotes SN material. The Sun is located at the origin, and the black solid line marks a distance of $1 \mathrm{AU}$

separated by a contact discontinuity, a structure similar to models of the heliosphere [16]. The contact discontinuity separates the solar wind from the supernova plasma, and is subject to Kelvin Helmholtz instabilities.

\subsection{Discussion}

\section{Dust Delivery}

This section summarizes work which will appear in Athanassiadou \& Fields [17].

\subsection{Motion of Charged Grains in the Solar System}

The dominant forces that act on macroscopic (a few microns) charged grains within the solar system are the gravitational force of the Sun, the radiation pressure of solar radiation and the Lorentz force due their motion through the interplanetary magnetic field (IMF). Consequently, their equation of motion is given by

$$
\ddot{\vec{x}}=(1-\beta) \frac{G M_{\odot}}{|\vec{x}|^{3}} \vec{x}-\frac{q}{m}\left(\dot{\vec{x}}-\vec{u}_{S W}\right) \times \vec{B}_{\odot}
$$

where $\beta$ is the strength of the radiation pressure expressed as the magnitude of the radiation pressure force to the magnitude of gravity, $G$ is the gravitational constant, $M_{\odot}$ is the mass of the Sun, $m$ 
is the charge of the grain, $q$ its mass, $\vec{u}_{S W}$ is the velocity of the solar wind and $\vec{B}_{\odot}$ is interplanetary magnetic field.

The interplanetary magnetic field, is given, to a good approximation by the Parker Interplanetary Magnetic Field [18], whose streamlines are an Archimedian spiral. To give a sense of the fields invovled, at the orbit of the Earth, the radial and azimuthal components are equal (the field line makes a $45^{\circ}$ with the tangent of the orbit) and have magnitude $B \sim 3 \mathrm{nT}$.

As a grain model, we adopt that of [19] where we assume the grains to be spherical particles of uniform density $\rho$, charge $q$ and radius $a$. If one further assumes they have a constant surface potential $U$ throughout their trajectories within the solar system, the charge of the grains will be given by $q=4 \pi \varepsilon_{0} U a$. The charge-to-mass ratio will then be given by $q / m=3 \varepsilon_{0} U / \rho a^{2}$.

We calculate the grain trajectories by solving Eq. (3.1) using a Runge-Kutta method of 4th order [20] in a 3-dimensions. The grains are placed $100 \mathrm{AU}$ away from the Sun either as individual grains, or a swarm of particles spanning a 5 AU square in a direction perpendicular to the grain's initial velocities. We then follow the grain trajectories while exploring the parameter space of initial velocities, voltage, and particle radius. In the paper we present results for grains of size $0.7 \mu \mathrm{m}$, a surface voltage of $5 \mathrm{~V}$ and initial velocities of 100,500 and $1000 \mathrm{~km} / \mathrm{s}$. The axis of symmetry of the magnetic field is at a 45 degree angle to the grain's initial velocity.

\subsection{Results}

The results of the above simulations are illustrated in Fig.3 and Fig 4. For grain incoming speeds of 500 and $1000 \mathrm{~km} / \mathrm{s}$, there is no deflection from a straight, ballistic trajectory, whereas the deflection for the grains traveling with $100 \mathrm{~km} / \mathrm{s}$ happens within a few AU from the scattering center (the Sun) and does not exceed 0.5 AU's. The trajectories, therefore of any grains traveling with speeds exceeding $100 \mathrm{~km} / \mathrm{s}$ are close to ballistic, and suffer little to no deflection within the computational volume.

\section{Conclusion}

A purely hydrodynamic interaction between a nearby supernova and the solar wind yields a limit of $10 \mathrm{pc}$ as the maximum distance the explosion could occur in order for supernova ejecta to be directly deposited on Earth. Such a distance is not consistent with the ${ }^{60} \mathrm{Fe}$ signal, and furthermore, such an event would have left imprints of biological damage which were not observed. On the other hand, supernova produced dust, if it survives within the remnant and gets within $100 \mathrm{AU}$ of the solar system with incoming speeds greater than $100 \mathrm{~km} / \mathrm{s}$, it would propagate within it almost ballistically (with minimal deflection). For such incoming speeds the maximum distance to the supernova could pushed back to $70 \mathrm{pc}$ for a supernova exploding into an average density interstellar medium $\left(\mathrm{n}=0.1 \mathrm{~cm}^{-3}\right)$. Therefore, not only it is more physical to assume that newly synthesized radioactivities are delivered in the form of dust grains, the dust delivery mechanism makes it easier and more probable.

Acknowledgments. TA would like to thank the organizers for generous financial support which made possible my attendance to this exciting conference. 

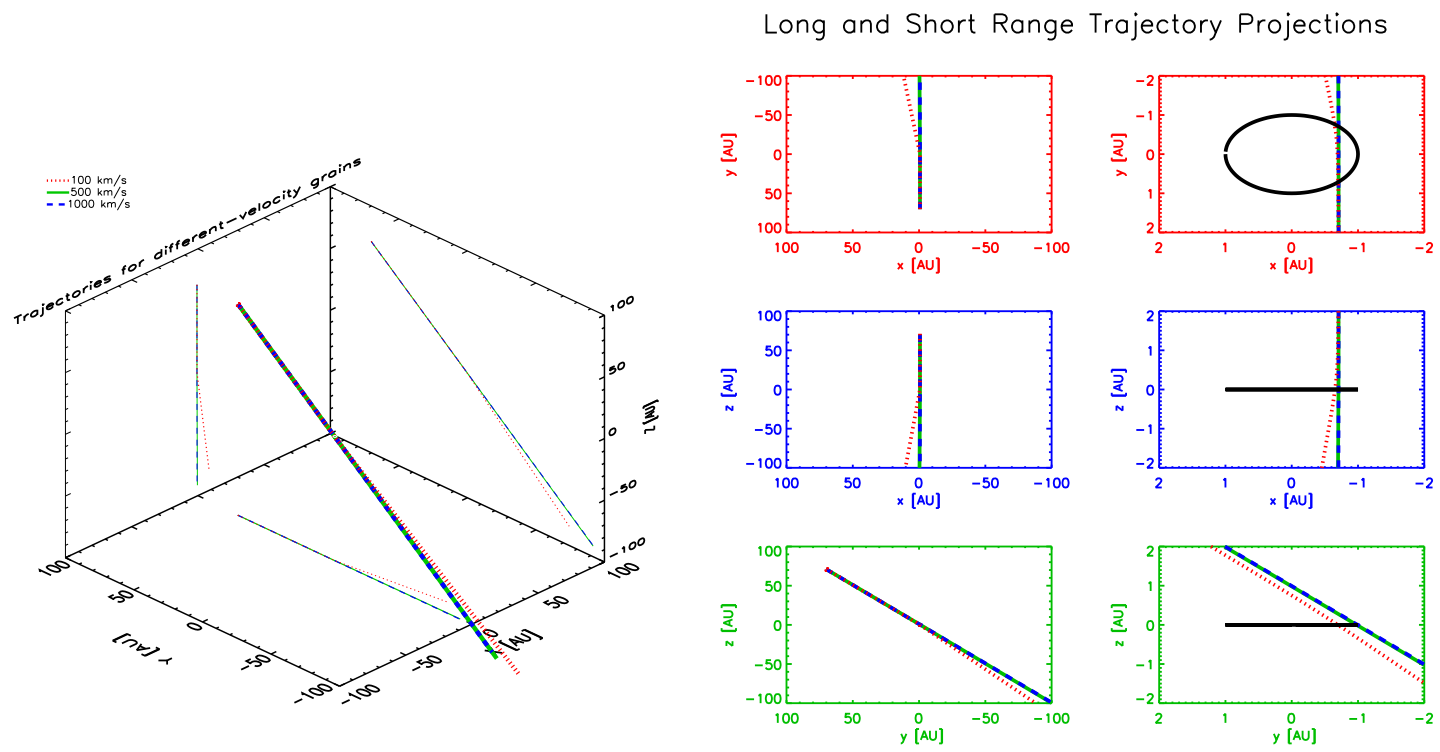

Figure 2: Trajectories for a grain of size $0.7 \mu \mathrm{m}$ with surface voltage $5 \mathrm{~V}$ and radiation-to-gravity strength of 0.46 [19]. The Sun is at the origin.
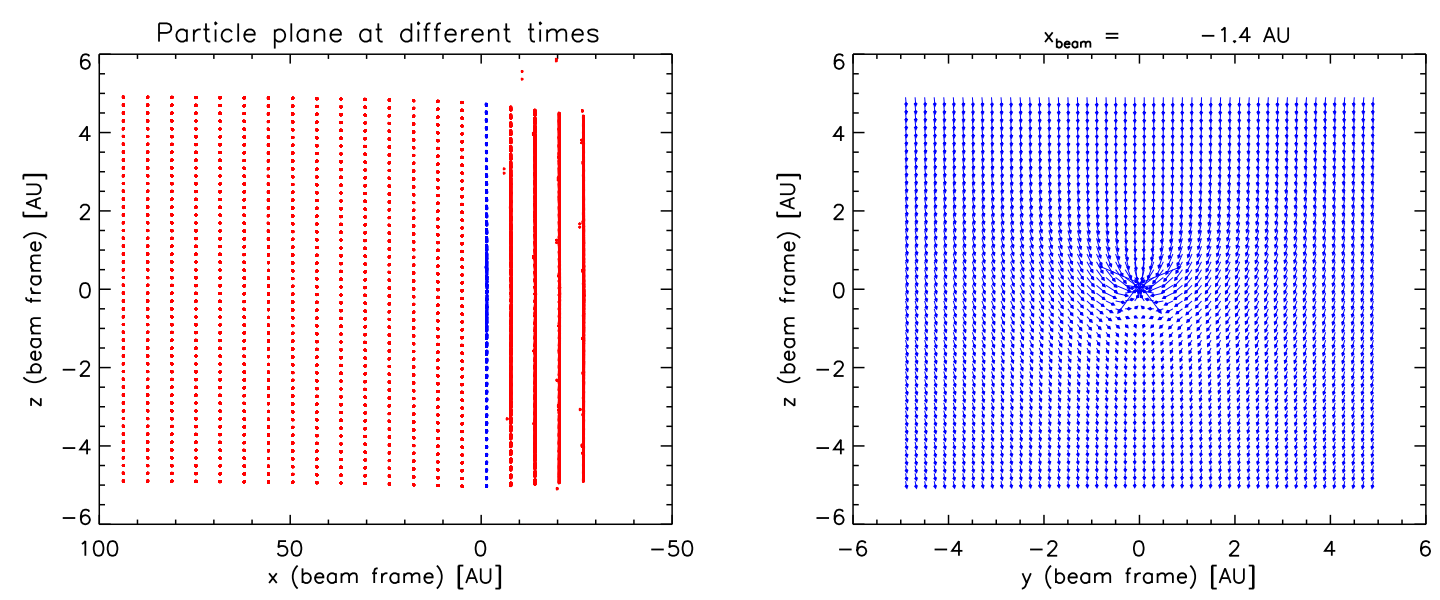

Figure 3: Trajectories for a plane of particles with initial speed $100 \mathrm{~km} / \mathrm{s}$

\section{References}

[1] Terry, K. D., \& Tucker, W. H. 1968, Science, 159, 421

[2] Laster, H., Tucker, W. H., \& Terry, K. D. 1968, Science, 160, 1138

[3] Benítez, N., Maíz-Apellániz, J., \& Canelles, M. 2002, Physical Review Letters, 88, 081101

[4] Ellis, J., Fields, B. D., \& Schramm, D. N. 1996, ApJ, 470, 1227

[5] Ruderman, M. A. 1974, Science, 184, 1079

[6] Gehrels, N., Laird, C. M., Jackman, C. H., Cannizzo, J. K., Mattson, B. J., \& Chen, W. 2003, ApJ, 585,1169 
[7] Knie, K., Korschinek, G., Faestermann, T., Wallner, C., Scholten, J., \& Hillebrandt, W. 1999, Physical Review Letters, 83, 18

[8] Knie, K., Korschinek, G., Faestermann, T., Dorfi, E. A., Rugel, G., \& Wallner, A. 2004, Physical Review Letters, 93, 171103

[9] Fields, B. D., Hochmuth, K. A., \& Ellis, J. 2005, ApJ, 621, 902

[10] Fields, B. D., Athanassiadou, T., \& Johnson, S. R. 2008, ApJ, 678, 549

[11] Sembach, K. R., \& Savage, B. D. 1996, ApJ, 457, 211

[12] Dwek, E. 1998, ApJ, 501, 643 and references therein

[13] Nittler, L. R., Amari, S., Zinner, E., Woosley, S. E., \& Lewis, R. S. 1996, ApJ Letters, 462, L31

[14] Nozawa, T., Kozasa, T., Habe, A., Dwek, E., Umeda, H., Tominaga, N., Maeda, K., \& Nomoto, K. 2007, ApJ, 666, 955

[15] Fryxell, B., et al. 2000, ApJ Supplement, 131, 273

[16] Zank, G. P. 1999, Space Science Reviews, 89, 413

[17] , in preparation 2008

[18] Parker, E. N. 1958, ApJ, 128, 664

[19] Landgraf, M. 2000, Journal of Geophysical Research, 105, 10303

[20] Press, W. H., Teukolsky, S. A., Vetterling, W. T., \& Flannery, B. P. 1992, Cambridge: University Press, |c1992, 2nd ed., 